\title{
Changes in soil physicochemical properties and soil bacterial community in alfalfa (Medicago sativa L.) / oat (Avena nuda L.) intercropping system
}

Xiaojing Liu ( $\square$ liuxj@gsau.edu.cn )

Gansu Agricultural University https://orcid.org/0000-0001-8800-4679

Yajiao Zhao

Gansu Agricultural University

Changchun Tong

Gansu Agricultural University

Yong Wu

Gansu Agricultural University

Research Article

Keywords: alfalfa/oat intercropping, rhizosphere soil, nutrients, bacterial community

Posted Date: April 7th, 2021

DOI: https://doi.org/10.21203/rs.3.rs-397347/v1

License: (c) (i) This work is licensed under a Creative Commons Attribution 4.0 International License. Read Full License 


\section{Abstract}

Aims Semi-arid region in northwest China is characterized by barren soil, high soil pH and high carbonate content.

Methods In order to improve the soil health status in this area, we studied the effects of alfalfa/oat intercropping on rhizosphere soil nutrients and bacterial community by Illumina MiSeq technology in the 3rd year.

Results The results showed that SOM significantly increased in the rhizosphere soil of intercropped compared with monoculture. AN, AP and AK of intercropping were significantly higher than those of monoculture of oat. Bacterial reads number, population taxonomic unit, richness and diversity all showed that intercropping were greater than monoculture. In terms of bacterial phylum, Proteobacteria, Bacteroidetes, Chloroflexi, Actinobacteria, Planctomycetes and Acidobacteria were dominant populations. The abundances of Proteobacteria, Acidobacteria, Cyanobacteria and Gemmatimonadetes were greater in intercropping systems than that in monoculture systems. At genus level, Opitutus, Pseudomonas, Gemmatimonas and Flavobacterium in intercropping were all higher than those in monoculture. At the same time, proteobacteria, bacteroidetes and actinobacteria were significantly positively correlated with SOM, TN and AN.

Conclusions This result indicated that alfalfa/oat intercropping can reduce the $\mathrm{pH}$ and significantly improve the microbial environment and nutrients of rhizosphere soil in northwest semi-arid region. Meanwhile, the rhizosphere soil microbial community and nutrients interact and regulate each other. Therefore, alfalfa/oat intercropping is an effective measure that improve soil environment in northwest semi-arid region.

\section{Introduction}

Soil plays a vital role in maintaining the balance of earth's ecosystem (Nesme et al, 2014). And soil is the habitat of various types of microorganisms (Hernández et al, 2015). Microorganisms play important roles in the biogeochemical cycle, particularly in the processes of nutrient cycling, system stability, antijamming capability, and sustainable development of soil (Jacoby et al, 2017). Soil microorganisms can decompose humus and organic matter in soil and transform them into forms that can be absorbed and utilized by plants, thus changing the content of soil nutrients. At the same time, soil nutrients provide the growing environment and energy for soil microorganisms, thus changing the number and community structure of soil microorganisms (Jonathan et al, 2018).

Intensive farming systems have been characterized as high input systems with chemical fertilizers, pesticides and simplified crop diversity, thus leading to potential problems regarding risks of soil degradation, environmental pollution, and low resilience. Soil microbial diversity and structure are likely to be affected by long-term continuous cropping of a single crop (Bulgarelli et al, 2013). Intercropping and crop rotation systems were reported to have positive impacts on soil microbial biomass and activity and populations of beneficial microorganisms such as the $\mathrm{N}$-fixing bacteria (Ding et al, 2013). Studies have shown that intercropping can indirectly affect soil microbial community composition and improve soil environment by changing plant physiological characteristics and root secretion characteristics (Li et al, 2007), and microbial number and community composition can also affect soil nutrients (Zhang et al, 2013). Previous work indicated that intercropping is able to maintain soil fertility in terms of soil organic matter (SOM) content, total N, Olsen P, exchangeable potassium (K) and soil enzyme activities (Wang et al, 2015). Use of legume/cereal intercropping system can be an especially sustainable and beneficial agricultural practice, as the $\mathrm{N}$-fixing crop provides natural $\mathrm{N}$ fertilizer for growing cereal. Intercropped legumes not only benefit from the symbiotic relationship of $\mathrm{N}$-fixing root nodules, but also exhibit improved $\mathrm{N}$ absorption efficiency (Santi et al, 2013). Plants have species-specific effects on soil microbial communities, and crop-based intercropping systems support a more diverse soil microbial community compared to conventional agricultural systems (Bainard et al, 2013).

The study area is located in the semi-arid region of northwest China, which has obvious regional characteristics such as drought, low vegetation coverage and fragile ecosystem. The land in this area is poor, with high soil pH and carbonate content and low organic matter content. It is easy to form saline-alkali land in the process of land use, which inhibits the activity of soil microorganisms and leads to soil nutrient shortage. Therefore, the combination of returning farmland to forest and grassland and efficient planting system is an effective method to prevent land degradation and to restore and improve soil environment. The semi-arid region in northwest China is the main producing area of herbage. Intercropping of legume and cereal forage can achieve the win-win result of production benefit and ecological protection. Alfalfa (Medicago sativa L.) is the highly valued legume forage because of its highest feeding value of all commonly grown hay crops (Li et al, 2017). Meanwhile, alfalfa can effectively fix $\mathrm{N}_{2}$ and improve the SOM and AN in the soil, which plays an important role in the enhancement of soil fertility (Yuan et al, 2016). Oat (Avena sativa L.) is one of the most important cereals cultivated in the research area. It is used animal nutrition, because it is appreciated for its nutritional value. Alfalfa/oat intercropping could significantly alleviate feed supply crisis and improve land utilization rate. Therefore, on the premise that alfalfa/maize intercropping can improve forage grass yield, this study hypothesized that alfalfa/maize intercropping can improve land utilization and improve soil environment. So, soil nutrients and bacterial community structure in rhizosphere were studied. It can provide a reference for maintaining the stability of the land ecosystem, and maintain the health of the soil microenvironment and improving the utilization efficiency of soil nutrient resources in the semi-arid region of northwest China.

\section{Materials And Methods}




\section{Experimental Materials}

Alfalfa, LW6010, was provided by college of Pratacultural Science, Gansu Agricultural University (GAU), China. Oat, Haywire, was provided by Beijing Clover Company, China. The rhizobium (Bradyrhizobium japonicum), Sinorhizobium meliloti (12531), was provided by GAU, China.

\section{Experimental Design}

The experiment was located at Forage Experimental Station Gansu Agricultural University ( $36^{\circ} 03^{\prime} \mathrm{N}, 103^{\circ} 40^{\prime} \mathrm{E}$ ), Lanzhou, Gansu Province, China. The local climate is temperate continental climate with more sunshine and dry climate. The mean annual temperature is $10.3^{\circ} \mathrm{C}$. The mean annual precipitation is $360 \mathrm{~mm}$. Most of the precipitation occurs between July, August and September with the unevenly distribution in the year. The mean annual elevation is $1410 \mathrm{~mm}$. The mean annual sunshine duration is $2600 \mathrm{~h}$. And the frost-free period is 180 days. The soil nutrients were as follows: pH 8.23; soil organic matter (SOM) $12.23 \mathrm{~g} \mathrm{~kg}^{-1}$; total nitrogen (TN) $0.81 \mathrm{~g} \mathrm{~kg}^{-1}$; available nitrogen (AN) $81.24 \mathrm{mg} \mathrm{kg}^{-1}$; available phosphorus (AP) $15.22 \mathrm{mg} \mathrm{kg}^{-1}$; available potassium (AK) $118.12 \mathrm{mg} \mathrm{kg}^{-1}$. The samples for the study described here were taken in 2016, before planted alfalfa and oat.

The experimental design consisted of three blocks (replications). Each block was divided into three plots representing the three plantation systems. Each plot unit comprised 24 rows ( $0.2 \mathrm{~m}$ per row) that were $5 \mathrm{~m}$ long and $4.8 \mathrm{~m}$ wide, each $24 \mathrm{~m}^{2}$ in size (Figure 1 ). Plots and blocks were separated from each other by $1 \mathrm{~m}$ walkways. Treatments levels included (1) alfalfa monoculture, (2) oat monoculture and (3) alfalfa intercropped with oat. For the intercropped treatment, 4 alfalfa rows were intercropped with 4 rows of oat. The ratio of alfalfa to oat was 1:1. The single cropping plots consisted of 24 rows of one plant species. Alfalfa seeds were soaked with rhizobia solution (OD600 was 0.63 0.64, soaked for $15 \mathrm{~min}$ ). Then it was planted in September 2016. Oat was planted in March 2017, 2018 and 2019. The sowing amount of alfalfa is $15 \mathrm{~kg} \mathrm{hm}^{-}$

2. The sowing amount of oat is $22.5 \mathrm{~kg} \mathrm{hm}^{-2}$. The phosphate fertilizer $\left(\mathrm{P}_{2} \mathrm{O}_{5}\right)$ and potash fertilizer $\left(\mathrm{K}_{2} \mathrm{O}\right)$ in each plot were applied before alfalfa sowing and greening. The application amount of phosphate fertilizer was $105 \mathrm{~kg} \mathrm{hm}^{2}$ and that of potassium fertilizer was $90 \mathrm{~kg} \mathrm{hm}^{2}$.

\section{Soil sampling}

The alfalfa and oat rhizosphere soils were randomly sampled by the 3-point sampling method in each plot of the monoculture systems and from the central rows in each plot of the intercropping systems by uplifting intact roots on June 2019 (alfalfa and oat generally reached early flowering stage). After shaking off the loosely adhered soils, the soils tightly adhering to root surface were brushed off and collected as the rhizosphere soil samples $(0 \sim 20 \mathrm{~cm})$. The rhizosphere soils obtained from the 3 plots were mixed and transported immediately to the laboratory. Each of the soil samples was sieved ( $<2 \mathrm{~mm}$ ) and then divided into two aliquots in sealed bags: one aliquot was stored at $-80^{\circ} \mathrm{C}$ for MiSeq sequencing of bacterial 16S rRNA gene (Sangon Biotech, Shanghai, China), and the other was determined after natural air drying for soil nutrients.

\section{Soil physicochemical properties}

The measurement methods of $\mathrm{pH}$ and soil nutrients were proposed by Jackson (1985). $\mathrm{pH}$ was measured by pH meter measurement (1:2.5 w/v) with a glass electrode. Soil organic matter (SOM) determined by wet oxidation using the acidified dichromate method. Total nitrogen (TN) was measured by kjeldahl method. Available nitrogen (AN) was measured by the alkaline hydrolysable method. Available phosphorus (AP) was measured by molybdenum blue method. Available kalium (AK) was extracted by incubating $5 \mathrm{~g}$ soil with $50 \mathrm{~mL}$ of $1 \mathrm{molL}^{-1} \mathrm{CH}_{3} \mathrm{COONH}_{4}\left(\mathrm{pH}^{7}\right)$ for $30 \mathrm{~min}$.

\section{Soil DNA extraction, PCR amplification, and MiSeq sequencing}

Total microbial community DNA was isolated from $1 \mathrm{~g}$ of soil per sample using an Omega Bio-Tek E.Z.N.A. Soil DNA extraction kit (Omega BioTek, Atlanta, GA, USA) according to the manufacturer's instructions. The extracted DNA was examined following electrophoresis in $1 \%$ agarose gel and the DNA was normalized to the same concentration prior to amplification. Extracted DNA from each sample was used as a template for amplification; the V4 V5 hypervariable region of the 16S rRNA gene was amplified three times using the primer F515: GTGCCAGCMGCCGCGG, and primer R907: CCGTCAATTCMTTTRAGTTT, with unique barcode sequence at the 5-end of each primer, respectively. The library quantification and pooling were measured. Finally, to dilute the PCR products of different samples based on request, a MiSeq library was constructed, followed by high-throughput sequencing.

\section{Statistical Analyses}

The raw data yielded from Illumina sequencing were analyzed using QIIME software. First, the effective sequences were filtered for decontamination, pruning, and removal of chimeric sequences. Then the optimized sequences were obtained. Operational Taxonomic Units (OTUs) were formed through cluster analysis, and then the OTUs at the similar level of $97 \%$ were statistically analyzed for biological information. 
OTUs and 16s DNA sequence numbers (Reads) were used to calculate the Alpha diversity, flora abundance index (Ace, Chao) and flora diversity index (Shannon, Simpson), and coverage of soil bacteria. Software platform mothur (version v.1.30.1) was used for index analysis.

Significant differences between treatments were assessed by ANOVA, using Duncan's multiple range tests. Data were subjected to two-way ANOVA test using cropping system and crop type as sources of variable. Based on OUT data, redundancy analysis was performed using Canoco for Windows 4.5, and Venn diagram, distance heatmap, redundancy analysis, and variation partition analysis were performed using R i386 3.2.3.

\section{Results}

\section{Physicochemical properties of hizosphere soil of alfalfa and oat}

Intercropping treatment extremely significant affected the contents of SOM and AP, and it significantly affected the contents of AN (Table 1). Crop type extremely significant affected the contents of SOM, TA, AN and AP, and it significantly affected pH. $\mathrm{pH}$ in intercropping had no significant difference than that in monoculture of alfalfa and oat. SOM significantly increased in the rhizosphere soil of intercropped alfalfa and oat. TN of MA was significantly higher than that of IA. AN and AP of MA and IA had no significant difference, but AN and AP of IO were significantly higher than those of MO. AK of MA was significantly higher than that of IA, and AK of IO was significantly higher than that of MO.

Table 1 Physicochemical parameters of different soil samples

\begin{tabular}{|lllllll|}
\hline Samples & $\mathrm{pH}$ & $\mathrm{SOM}$ & $\mathrm{TN}$ & $\mathrm{AN}$ & $\mathrm{AP}$ & $\mathrm{AK}$ \\
& & $\mathrm{g} \cdot \mathrm{kg}^{-1}$ & $\mathrm{~g} \cdot \mathrm{kg}^{-1}$ & $\mathrm{mg} \cdot \mathrm{kg}^{-1}$ & $\mathrm{mg} \cdot \mathrm{kg}^{-1}$ & $\mathrm{mg} \cdot \mathrm{kg}^{-1}$ \\
\hline MA & $7.73 \pm 0.04^{\mathrm{ab}}$ & $13.21 \pm 0.07^{\mathrm{b}}$ & $0.79 \pm 0.02^{\mathrm{a}}$ & $80.68 \pm 1.88^{\mathrm{a}}$ & $14.39 \pm 0.63^{\mathrm{c}}$ & $95.60 \pm 3.11^{\mathrm{a}}$ \\
\hline IA & $7.67 \pm 0.06^{\mathrm{b}}$ & $13.55 \pm 0.20^{\mathrm{a}}$ & $0.76 \pm 0.02^{\mathrm{b}}$ & $82.17 \pm 1.35^{\mathrm{a}}$ & $14.54 \pm 0.59^{\mathrm{bc}}$ & $87.94 \pm 1.62^{\mathrm{b}}$ \\
\hline MO & $7.80 \pm 0.05^{\mathrm{a}}$ & $10.86 \pm 0.20^{\mathrm{d}}$ & $0.70 \pm 0.03^{\mathrm{c}}$ & $65.92 \pm 1.45^{\mathrm{c}}$ & $15.45 \pm 0.18^{\mathrm{b}}$ & $90.62 \pm 1.19^{\mathrm{b}}$ \\
\hline IO & $7.75 \pm 0.06^{\mathrm{ab}}$ & $11.87 \pm 0.19^{\mathrm{c}}$ & $0.72 \pm 0.01^{\mathrm{c}}$ & $68.66 \pm 0.79^{\mathrm{b}}$ & $17.56 \pm 0.46^{\mathrm{a}}$ & $95.78 \pm 0.94^{\mathrm{a}}$ \\
\hline Results of two-way ANOVA test & & & & \\
\hline Int. & 0.105 & $0.000^{* *}$ & 0.635 & $0.033^{*}$ & $0.004^{* *}$ & 0.287 \\
\hline Crt. & $0.026^{*}$ & $0.000^{* *}$ & $0.000^{* *}$ & $0.000^{* *}$ & $0.000^{* *}$ & 0.232 \\
\hline
\end{tabular}

Note: Values are means \pm standard deviation $(n=3)$. Different lowercase letters meant significant differences among different samples according to the Duncan's multiple range test $(P<0.05)$. SOM, TN, AN, AP, and AK represent organic matter, total nitrogen, available nitrogen, available phosphorus and available potassium. MA, IA, MO, and IO represent monoculture alfalfa, intercropped alfalfa, monoculture oat, and intercropped oat, respectively. Int. and Crt. represent intercropping treatment and crop type, respectively. Here, * and ** represent significance at 0.05 and 0.01 levels. The same abbreviations appear below.

\section{Microbial richness and diversity}

A total of 610,796 sequences were obtained from the 4 soil samples by MiSeq analysis of the V3 V4 region of bacterial $16 S$ rRNA genes (Table 2). Intercropping treatment extremely significant affected ACE, and it significantly affected sequences. Crop type extremely significant affected Shannon index and Simpson index, and it significantly affected $\mathrm{pH}$. The optimized sequence numbers for each sample ranged from 145,882 to 164,599. Among all the samples, a total of 19,933 OTUs were obtained, ranging from 4751 in monoculture soil to 5085 in intercropping rhizosphere soil. The coverage index of each sample was estimated to be up to $99 \%$. The richness and diversity estimators (Chao1, ACE and Shannon) of intercropping alfalfa and oat rhizosphere soils were higher than those of the corresponding monoculture rhizosphere soils. Simpson index of monoculture alfalfa and oat rhizosphere soils were higher than those of the corresponding intercropping rhizosphere soils.

Table 2 Bacterial richness and diversity of MA, IA, MO, and IO 


\begin{tabular}{|llllllll|}
\hline \multirow{2}{*}{ Samples } & \multicolumn{5}{l}{ Sequencing results } & \multicolumn{5}{l|}{ Richness and diversity estimates } \\
\cline { 2 - 8 } & Sequences & OUT & Coverage index & Chao1 index & ACE & Shannon index & Simpson index \\
\hline MA & $145882 \pm 951^{\mathrm{b}}$ & $4751 \pm 216^{\mathrm{a}}$ & $0.99 \pm 0.0032^{\mathrm{a}}$ & $5642 \pm 30^{\mathrm{a}}$ & $5599 \pm 64^{\mathrm{b}}$ & $6.66 \pm 0.03^{\mathrm{b}}$ & $0.0051 \pm 0.0002^{\mathrm{a}}$ \\
\hline IA & $150348 \pm 4039^{\mathrm{b}}$ & $5016 \pm 76^{\mathrm{a}}$ & $0.99 \pm 0.0041^{\mathrm{a}}$ & $5872 \pm 188^{\mathrm{a}}$ & $5839 \pm 43^{\mathrm{a}}$ & $6.76 \pm 0.14^{\mathrm{b}}$ & $0.0052 \pm 0.0002^{\mathrm{a}}$ \\
\hline MO & $149967 \pm 858^{\mathrm{b}}$ & $5081 \pm 47^{\mathrm{a}}$ & $0.99 \pm 0.0036^{\mathrm{a}}$ & $5753 \pm 70^{\mathrm{a}}$ & $5715 \pm 16^{\mathrm{ab}}$ & $7.02 \pm 0.06^{\mathrm{ab}}$ & $0.0023 \pm 0.0001^{\mathrm{b}}$ \\
\hline IO & $164599 \pm 1813^{\mathrm{a}}$ & $5085 \pm 44^{\mathrm{a}}$ & $0.99 \pm 0.0034^{\mathrm{a}}$ & $5823 \pm 47^{\mathrm{a}}$ & $5812 \pm 44^{\mathrm{a}}$ & $7.05 \pm 0.02^{\mathrm{a}}$ & $0.0024 \pm 0.0001^{\mathrm{b}}$ \\
\hline Results of two-way ANOVA test & & & & & \\
\hline Int. & $0.011^{*}$ & 0.260 & 1.000 & 0.196 & $0.005^{* *}$ & 0.921 & 0.715 \\
\hline Crt. & $0.013^{*}$ & 0.110 & 1.000 & 0.781 & 0.337 & $0.007^{* *}$ & $0.000^{* *}$ \\
\hline
\end{tabular}

Note囚a-Species level at $97 \%$ similarity threshold is defined as one operational taxonomic unit (OUT). MA, alfalfa monoculture; IA, intercropping alfalfa; MO, oat monoculture; $\mathrm{IO}$, intercropping oat.

\section{Shared bacterial OTUs}

Venn diagrams revealed the total observed OTUs in soil samples (Fig.2), and 19,934 OTUs were common to all soil samples. Moreover, the distribution of sequences also demonstrated that each plant rhizosphere had its own microbial population.

\section{Variation of OTU abundance at phyla level}

The dominant bacterial phyla were Proteobacteria, Bacteroidetes, Chloroflexi, Actinobacteria, Planctomycetes, Acidobacteria, Verrucomicrobia, Cyanobacteria, Gemmatimonadetes, Firmicutes, Thaumarchaeota and Nitrospirae (Fig. 3). Other sequences belonged to Unassigned, No Rank and others were always found in very low proportions. Comparative analysis of the four soil samples revealed a distinct distribution of the bacterial phyla. On average, Actinobacteria showed a higher relative abundance in alfalfa rhizosphere soils than in oat rhizosphere soils, whereas Proteobacteria, Chloroflexi, Acidobacteria, Cyanobacteria, Thaumarchaeota and Nitrospirae showed the opposite pattern. Thaumarchaeota in monoculture (MA and MO) was significantly higher than that in intercropping (IA and IO). However, Chloroflexi showed an opposite pattern to the one observed for Thaumarchaeota, which Chloroflexi showed a higher abundance in intercropping libraries than monoculture libraries. Furthermore, in alfalfa soil, Bacteroidetes, Actinobacteria, and Firmicutes showed a significant higher abundance in intercropping than that in monoculture, whereas Chloroflexi and Verrucomicrobia showed a significant higher abundance in monoculture than that in intercropping. In oat soil, Chloroflexi and Planctomycetes presented the dominant phyla in the intercropping treatment; Actinobacteria, Gemmatimonadetes and Firmicutes presented disadvantages in intercropping treatment.

\section{Variation of OTU abundance at genus level}

Hierarchically clustered heatmap analysis, based on the microbial community profiles at the genus level, was used to identify the different compositions of the microbial community (Fig. 4). The IA and MA groups were separated from IO and MO groups, suggesting the clear distinction of microbial community composition between alfalfa and oat groups. At the genus level, comparison of the relative abundances revealed significant differences between monoculture and intercropping soil bacterial communities. The distribution of the dominant genera varied significantly between monoculture and intercropping soils. Flavobacterium (5.10\%), Gp6 (2.46\%), Cellvibrio (1.72\%), Ohtaekwangia (1.68\%), Pseudomonas (1.32\%), Gemmatimonas (1.29\%), Opitutus (1.59\%), Streptophyta (1.91\%) and Arenimonas (0.92\%) showed a higher relative abundance in alfalfa intercropping soils than in corresponding monoculture soils, whereas Algoriphagus (2.11\%), Chryseolinea (1.10\%), Geobacter $(0.71 \%)$, Pedobacter ( $0.40 \%)$, Devosia ( $0.39 \%)$ and Streptomyces $(0.24 \%)$ showed the opposite pattern (Fig. 5). Flavobacterium (3.11\%), Gp6 (2.91\%), Pseudomonas (2.77\%), Gemmatimonas (2.00\%), Ohtaekwangia (1.71\%), Opitutus (1.48\%), Algoriphagus (1.36\%), Gp7 (1.17\%), Hydrogenophaga (0.91\%), Bacillariophyta $(1.10 \%)$ and Arthrobacter $(0.98 \%)$ were present in higher proportions in oat intercropping soils compared with corresponding monoculture soils. Compared with intercropping and monoculture at the genus level, Opitutus, Pseudomonas, Gemmatimonas and Flavobacterium in intercropping were all higher than those in monoculture. Pedobacter and Streptomyces in intercropping were lower than those in monoculture. Algoriphagus in intercropping was higher than that in monoculture of alfalfa; however it in intercropping of oat was lower than that in monoculture of oat.

\section{Correlations of environmental data and bacterial communities Mantel tests}

To investigate relationships between soil microbial community composition and measured environmental variables, different bacterial phyla were analysed using Redundancy Analysis (RDA) (Fig. 5). Overall, the two RDA axes explained $93.67 \%$ of the variation between the soil bacterial 
communities. The distinctions of bacterial community structure among alfalfa and oat groups were also supported by RDA. Oat samples were clustered together and were well separated from that alfalfa samples. AK had the longest arrow, indicating that it was most important in influencing the bacterial community. $\mathrm{PH}$ and TN were smaller in influencing the bacterial community.

The abundances of Proteobacteria was significantly negatively correlated with $\mathrm{pH}$, and it was significantly positive correlated with SOM, TN and AN. Chloroflexi showed the opposite trend. Bacteroidetes was significantly positive correlated with TN, AN and AP. The abundance of Actinobacteria had a markedly positive relationship with SOM, TN and AN. Planctomycetes was significantly negatively correlated with TN and AN. Acidobacteria was significantly negatively correlated with AK. Verrucomicrobia was significantly negatively correlated with TN and AK.

TABLE 3 Pearson's correlation ( $r$ ) and significance $(p)$ values between the abundances of the dominant abundant bacterial phyla and the soil variables

\begin{tabular}{|c|c|c|c|c|c|c|c|c|c|c|c|c|}
\hline & $\mathrm{pH}$ & & SOM & & $\mathrm{TN}$ & & AN & & AP & & AK & \\
\hline & $r$ & $\mathrm{p}$ & $r$ & $\mathrm{p}$ & $r$ & $\mathrm{p}$ & $r$ & $\mathrm{p}$ & $r$ & $\mathrm{p}$ & $r$ & $\mathrm{p}$ \\
\hline Proteobacteria & -0.61 & 0.036 & 0.70 & 0.012 & 0.42 & 0.178 & 0.59 & 0.042 & -0.07 & 0.824 & -0.01 & 0.967 \\
\hline Bacteroidetes & -0.24 & 0.449 & 0.57 & 0.054 & 0.82 & 0.001 & 0.64 & 0.026 & -0.6 & 0.041 & 0.3 & 0.341 \\
\hline Chloroflexi & 0.63 & 0.030 & -0.71 & 0.010 & -0.75 & 0.005 & -0.7 & 0.012 & 0.42 & 0.175 & -0.31 & 0.330 \\
\hline Actinobacteria & -0.49 & 0.105 & 0.86 & 0.000 & 0.86 & 0.000 & 0.84 & 0.001 & -0.47 & 0.125 & 0.33 & 0.290 \\
\hline Planctomycetes & 0.33 & 0.294 & -0.57 & 0.053 & -0.69 & 0.013 & -0.68 & 0.016 & 0.76 & 0.004 & 0.19 & 0.563 \\
\hline Acidobacteria & -0.15 & 0.646 & -0.11 & 0.734 & -0.25 & 0.435 & -0.01 & 0.980 & -0.38 & 0.225 & -0.62 & 0.033 \\
\hline Verrucomicrobia & 0.26 & 0.418 & -0.34 & 0.277 & -0.61 & 0.035 & -0.38 & 0.217 & 0.31 & 0.322 & -0.61 & 0.037 \\
\hline
\end{tabular}

Note: Values in bold indicate significant correlations $(p<.01)$.

\section{Discussions}

\section{Soil physicochemical properties}

The $\mathrm{pH}$ of rhizosphere soil after planting was significantly lower than that before planting, while that in intercropping was lower than that in monoculture of alfalfa and oat. This is because that root interaction in intercropping system can promote the production of organic acids to dissociate $\mathrm{H}^{+}$( $\mathrm{Li}$ et al, 2007). At the same time, intercropping can make legume crops release $\mathrm{H}^{+}$in the process of $\mathrm{N}$ fixation. In addition, intercropping can enhance soil phosphatase activity and promote the release of $\mathrm{H}^{+}$in the dephosphorization reaction of organophosphorus, thereby intercropping can reduce the soil $\mathrm{pH}$ value. Latati et al. (2014) also found that under alkaline conditions, both maize/cowpea intercropping and single cowpea planting reduced the $\mathrm{pH}$ of rhizosphere soil, and the change of intercropping was larger than that of monoculture. SOM content in intercropping of rhizosphere soil was significantly increased than that in monoculture of alfalfa and oat. Soil SOM plays an important role in almost soil function. Alfalfa as perennial pasture reduces the number of soil tillage disturbances and thus increases the transformation of humus. SOM is decomposed by microbes. The changes of the kinds and quantities of the microbes that take part in the degradation of the soil organic matter can indirectly affect the content of SOM in soil. Fornara and Tilman also found that high-diversity grass mixtures stored more soil $\mathrm{C}$ than did monocultures (Fornara et al, 2008). So, the intercropping system was beneficial to the availability of rhizosphere soil SOM.

AN, AP and AK content in the intercropping were significantly higher than that in monoculture of oat rhizosphere soil. Intercropping can promote the nodule and $\mathrm{N}$ fixation of alfalfa, increase the content of $\mathrm{AN}$, and reduce the use of TN in rhizosphere soil. Therefore, the content of TN of intercropped alfalfa is lesser than that of monoculture. At the same time, oat has more competitive nutrient ability than alfalfa. Previous studies showed a common result that $\mathrm{N}$ fixation capacity of legumes may be enhanced by intercropping when the nonlegume is a strong competitor for soil inorganic nitrogen (Karpenstein-Machan et al, 2000). So, there is no significant difference in AN of alfalfa rhizosphere soil between intercropping and monoculture. Intercropping affected soil phosphatase, catalase, organic acids and phenolic acids et al., thus promoting the activation and transformation of AP, while the activation and decomposition accumulation AK in soil was relatively slow. Oat was more competitive with AP and AK than alfalfa (Wang et al, 2017). Therefore, AP was no significance in intercropping and monoculture of alfalfa, AK in intercropping was significantly lower than that in monoculture of alfalfa. Oats continuously absorb AP and AK from alfalfa rhizosphere soil, so that AP and AK in intercropping of oat are greater than those in monocultures. Similar results were observed in other legume and cereal intercropping systems (Li et al, 2004; Xia et al, 2013).

\section{Bacterial community diversity and structure}


The major factor affecting soil microbial diversity is land-use type including monoculture, long-term continuous cropping, and intercropping (Buyer et al, 2010). Soil microbial diversity is important for soil functions, including mineral nutrient cycling, organic matter turnover, and soil structure formation (Jangid et al, 2011). In particular, soil microbial diversity is critical for maintaining soil health, which is one of the most important factors for plant production in agricultural systems. The analysis on richness estimators (Ace and Chao 1) and the diversity indices (Shannon and Simpson) revealed a richer bacterial community in intercropping than that of monoculture rhizosphere soils. Several studies have reported that soil bacterial diversity could be affected by many factors, including soil fertility, vegetation, planting method, type of crop, and plant age (Epelde et al, 2015). The bacterial community structure in alfalfa or oat rhizosphere soils were different between the monoculture and intercropping system. Li et al. (2016) believe that this phenomenon is caused by the intercropping between the roots of two crops, and the changes in the composition and content of root exudes lead to changes in the flora structure of soil microorganisms, thus affecting the quantity and diversity of soil microorganisms in the rhizosphere. In all samples, the dominant taxonomic groups were Proteobacteria, Bacteroidetes, Chloroflexi, Actinobacteria, Planctomycetes and Acidobacteria. These phyla have been depicted as common inhabitants of rhizosphere soil (Caporaso et al, 2012). The abundances of Proteobacteria, Acidobacteria, Cyanobacteria and Gemmatimonadetes were greater in intercropping systems than that in monoculture systems. It indicated that intercropping could promote the growth of Proteobacteria, Acidobacteria, Cyanobacteria and Gemmatimonadetes. At genus level, Flavobacterium, Algoriphagus, Gp6, Ohtaekwangia, Pseudomonas, Gemmatimonas, Opitutus and Chryseolinea were the most abundance genera across all soil samples. This indicated these genera might be indigenous in soil sampled. Opitutus, Pseudomonas, Gemmatimonas and Flavobacterium in intercropping were all higher than those in monoculture. It indicates that they are the dominant bacteria in intercropping that can improve soil environment.

\section{Effects of soil properties and planting pattern on the dominant bacterial phyla and bacterial community structure}

Microorganisms are the main decomposers and the most active factors in soil. Soil nutrients also provide the environment and energy for microbial life. Redundancy analysis (RDA) showed that alfalfa and oat were separated through the RDA component 1, and the intercropping and monoculture were separated by the RDA component 2. Intercropping may affect soil physicochemical properties indirectly through plant-mediate changes in soil microbial community (Jacoby et al, 2017). Previous studies have demonstrated that plants can modify their rhizosphere microbial community through their root exudates, and that the shift of rhizosphere microbial can further affect the soil enzymes and soil fertility. Therefore, the rhizosphere microbial community can be affected by different plants in intercropping, and the changes in soil microbial communities may play important roles in the benefits of intercropping. In this study, proteobacteria, bacteroidetes and actinobacteria were significantly positively correlated with SOM, TN and AN. Other studies have shown that the abundance of proteobacteria is related to the use of carbon, and the change of SOM is 7/highly correlated with the abundance of proteobacteria (Fierer et al, 2007). Bacteroidetes are the main mineralizers of organic carbon ( $\mathrm{Li}$ et al, 2011), so bacteroidetes can increase the content of organic carbon, provide energy for other microorganisms and soil enzyme activities, and affect the content of other nutrients. Actinomycetes are involved in SOM transformation. So, the abundance of actinomycetes can reflect the amount of SOM. Therefore, the abundance of proteobacteria, bacteroidetes and actinobacteria determines the level of soil nutrients, which also indicates that intercropping can promote the growth of beneficial bacteria.

\section{Conclusions}

Alfalfa/oat intercropping can reduce the $\mathrm{pH}$ value and significantly improve the microbial environment and nutrients of rhizosphere soil in northwest semi-arid region. Meanwhile, the rhizosphere soil microbial community and nutrients interact and regulate each other. Therefore, alfalfa/oat intercropping is an effective measure that improve soil environment in northwest semi-arid region.

\section{Declarations}

\section{AUTHOR CONTRIBUTIONS}

$\mathrm{XL}$ took part in designing the experiment and participated in drafting the work; YZ conceived and designed the experiment, conducted it and acquired the data, analyzed the data and wrote the manuscript; CT and YW helped experiment test.

\section{FUNDING}

This work was supported by National Natural Science Foundation of China (31460622) and special funds for discipline construction (GAU-XKJS2018-008).

\section{ACKNOWLEDGMENTS}

The authors thank Weibiao Liao at Gansu Agricultural University for his suggestions and English correction. The authors also thank a reviewer for suggesting the principle component analysis.

\section{References}


Bainard, L., Koch, A., Gordon, A., Klironomos, J. (2013). Growth response of crops to soil microbial communities from conventional monocropping and tree-based intercropping systems. Plant Soil, 363(1-2): 345-356. doi: 10.1007/s11104-012-1321-5

Buyer, J. S., Teasdale, J. R., Roberts, D. P., Zasada, I. A. (2010). Factors affecting soil microbial community structure in tomato cropping systems. Soil Biol. Biochem., 42(5): 831-841. doi: 10.1016/j.soilbio.2010.01.020.

Caporaso, J. G., Lauber, C. L., Walters, W. A., Berg-Lyons, D., Huntley, J., Fierer, N., Owens, . M., Betley, J., Fraser, L., Bauer, M., Gormley, N., Gilbert, J. A., Smith, G., Knight, R. (2012). Ultra-high-throughput microbial community analysis on the Illumina HiSeq and MiSeq platforms. ISME J., 6(8): 1621-1624. doi: 10.1038/ismej.2012.8.

Epelde, L., Lanzen, A., Blanco, F., Urich, T. (2015). Adaptation of soil microbial community structure and function to chronic metal contamination at an abandoned Pb-Zn mine. FEMS Microbiol. Ecol., 91(1): 1-11. doi: 10.1093/femsec/fiu007.

Fierer, N., Bradford, M. A., Jackson, R. B. Toward an ecological classification of soil bacteria. Ecology, 2007, 88(6): 1354-1364. doi: 10.1890/051839.

Fornara, D., Tilman, D. (2008). Plant functional composition influences rates of soil carbon and nitrogen accumulation. J. Ecol., 96(6): 314-322. doi: 10.1111/j.1365-2745.2007.01345.x.

Jackson, M.L. Soil Chemical Analysis. Prentice-Hall: Upper Saddle River, N J, USA, 1958.

Jacoby, R., Peukert, M., Succurro, A., Koprivova, A., Kopriva, S. (2017). The Role of soil microorganisms in plant mineral nutrition-current knowledge and future directions. Front. Plant Sci., 8. doi: 10.3389/fpls.2017.01617

Jangid, K., Williams, M. A., Franzluebbers, A. J., Schmidt, T. M., Coleman, D. C., \& Whitman, W. B. (2011). Land-use history has a stronger impact on soil microbial community composition than aboveground vegetation and soil properties. Soil Biol. Biochem., 43(10): 2184-2193. doi: 10.1016/j.soilbio.2011.06.022.

Karpenstein-Machan, M., \& Stuelpnagel, R. (2000). Biomass yield and nitrogen fixation of legumes monocropped and intercropped with rye and rotation effects on a subsequent maize crop. Plant Soil, 218(2): 215-232. doi: 10.1023/a:1014932004926

Latati, M., Blavet, D., Alkama, N., Laoufi, H., Drevon, J. J., Gérard, F., Pansu, M., Ounane, S. M. (2014). The intercropping cowpea-maize improves soil phosphorus availability and maize yields in an alkaline soil. Plant Soil, 385(1-2): 1-11. doi: 10.1007/s11104-014-2214-6.

Li, L., Li, S. M., Sun, J. H., Zhou, L. L., Bao, X. G., Zhang, H. G., Zhang, F. S. (2007) Diversity enhances agricultural productivity via rhizosphere phosphorus facilitation on phosphorus-deficient soils. P. NT. Acad. Sci., 104(27): 11192-11196. doi: 10.1073/pnas.0704591104.

Li, Q. S., Wu, L. K., Chen, J., Khan, M. A., Luo, X., Lin, W. (2016). Biochemical and microbial properties of rhizospheres under maize /peanut intercropping. J. Inter. Agr., 15(1): 101-110. doi: 10.1016/S2095-3119(15)61089-9.

Li, S., Li, L., Zhang, F. S., Tang, C. (2004). Acid phosphatase role in chickpea/maize intercropping. Ann. Botan., 94(2): 297-303. doi: $10.1093 / a o b / m c h 140$.

Li, Y., Lee, C. G., Watanabe, T., Murase, J., Asakawa, S., Kimura, M. (2011). Identification of microbial communities that assimilate substrate from root cap cells in an aerobic soil using a DNA-SIP approach. Soil Biology Biochem., 43(9): 1928-1935. doi: 10.1016/j.soilbio.2011.05.016.

Li, Z., Long, R., Zhang, T., Wang, Z., Zhang, F., Yang, Q., Sun, Y. (2017). Molecular cloning and functional analysis of the drought tolerance gene MsHSP70 from alfalfa (Medicago sativa L.). J. Plant Res., 130(2): 387-396. doi:10.1007/s10265-017-0905-9

M. Hernández, M.G. Dumont, Q. Yuan, R. Conrad, Different Bacterial Populations Associated with the Roots and Rhizosphere of Rice Incorporate Plant-Derived Carbon. Appl. Environ. Microbiol. 81 (60) (2015) 6 2244-2253.

Nesme, T., Colomb, B., Hinsinger, P., \& Watson, C. A. (2014). Soil phosphorus management in organic cropping systems: from current practices to avenues for a more efficient Use of P resources. Org. Farming, Prototype Sustain. Agri., 23-45. doi:10.1007/978-94-007-7927-3_2

Santi, C., Bogusz, D., Franche, C. (2013). Biological nitrogen fixation in nonlegume plants. Ann Bot, 111(5): $743-767$.

Wang, X., Deng, X., Pu, T., Song, C., Yong, T. W., Yang F, Sun, X., Liu, W. G., Yan, Y. H., Du, J. B., Liu, J., Su, K., Yang, W. Y. (2017). Contribution of interspecific interactions and phosphorus application to increasing soil phosphorus availability in relay intercropping systems. Field Crop. Res., 204: 12-22. doi: 10.1016/j.fcr.2016.12.020. 
Wang, Z. G., Bao, X. G., Li, X. F., Jin, X., Zhao, J. H., Sun, J. H., Christie, P., Li, L. (2015). Intercropping maintains soil fertility in terms of chemical properties and enzyme activities on a timescale of one decade. Plant Soil, 391(1-2): 265-282. doi: 10.1007/s11104-015-2428-2.

Xia, H. Y., Wang, Z. G., Zhao, J. H., Sun, J. H., Bao, X.-G., Christie, P., Li, L. (2013). Contribution of interspecific interactions and phosphorus application to sustainable and productive intercropping systems. Field Crop. Res, 154: 53-64. doi:10.1016/j.fcr.2013.07.011.

Yuan, Z. Q., Yu, K. L., Epstein, H., Fang, C., Li, J. T., Liu, Q.-Q., Li, F.M. (2016). Effects of legume species introduction on vegetation and soil nutrient development on abandoned croplands in a semi-arid environment on the Loess Plateau, China. Sci. Total Environ., 541: 692-700. doi:10.1016/j.scitotenv.2015.09.108.

\section{Figures}
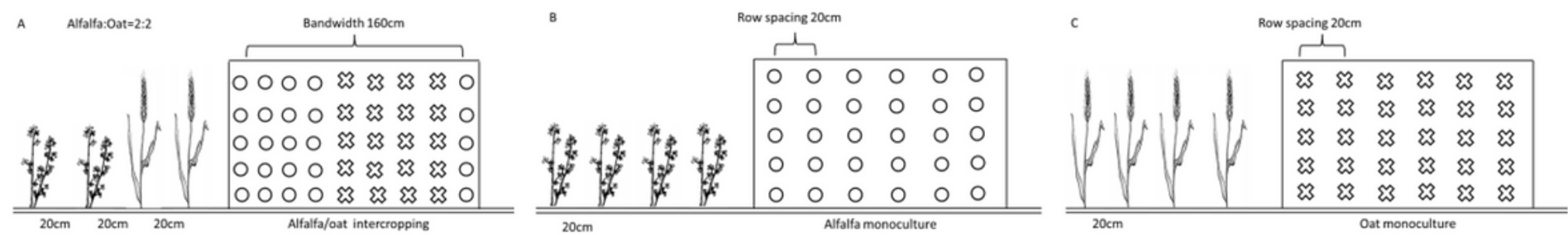

\section{Figure 1}

Diagram of planting system

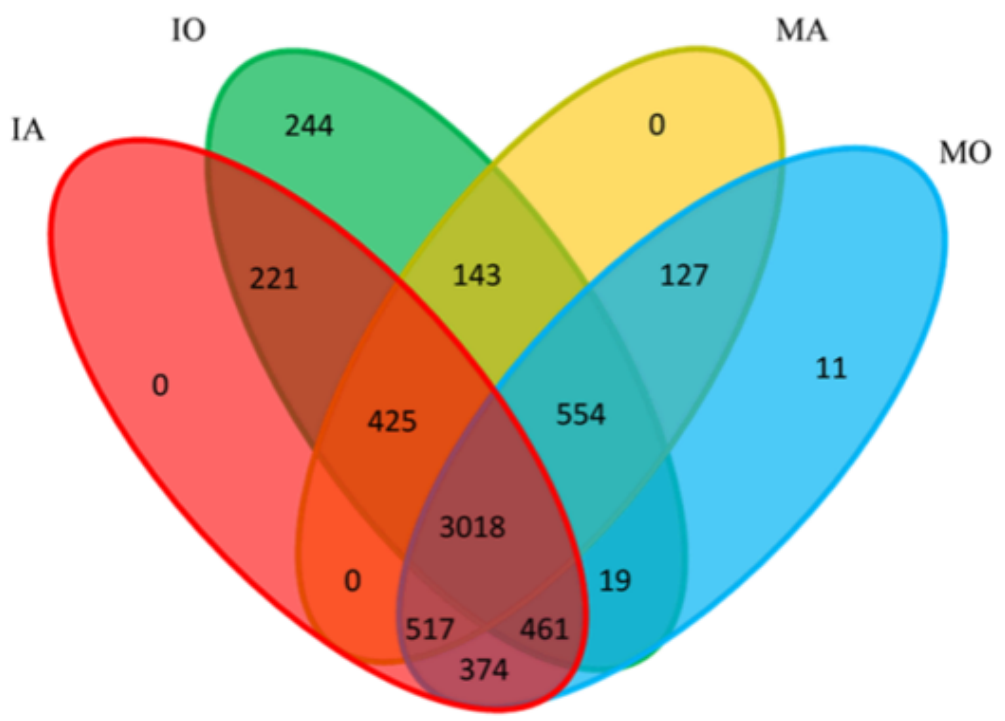

Figure 2

Venn diagram showing the shared bacterial OTUs in all soil samples 


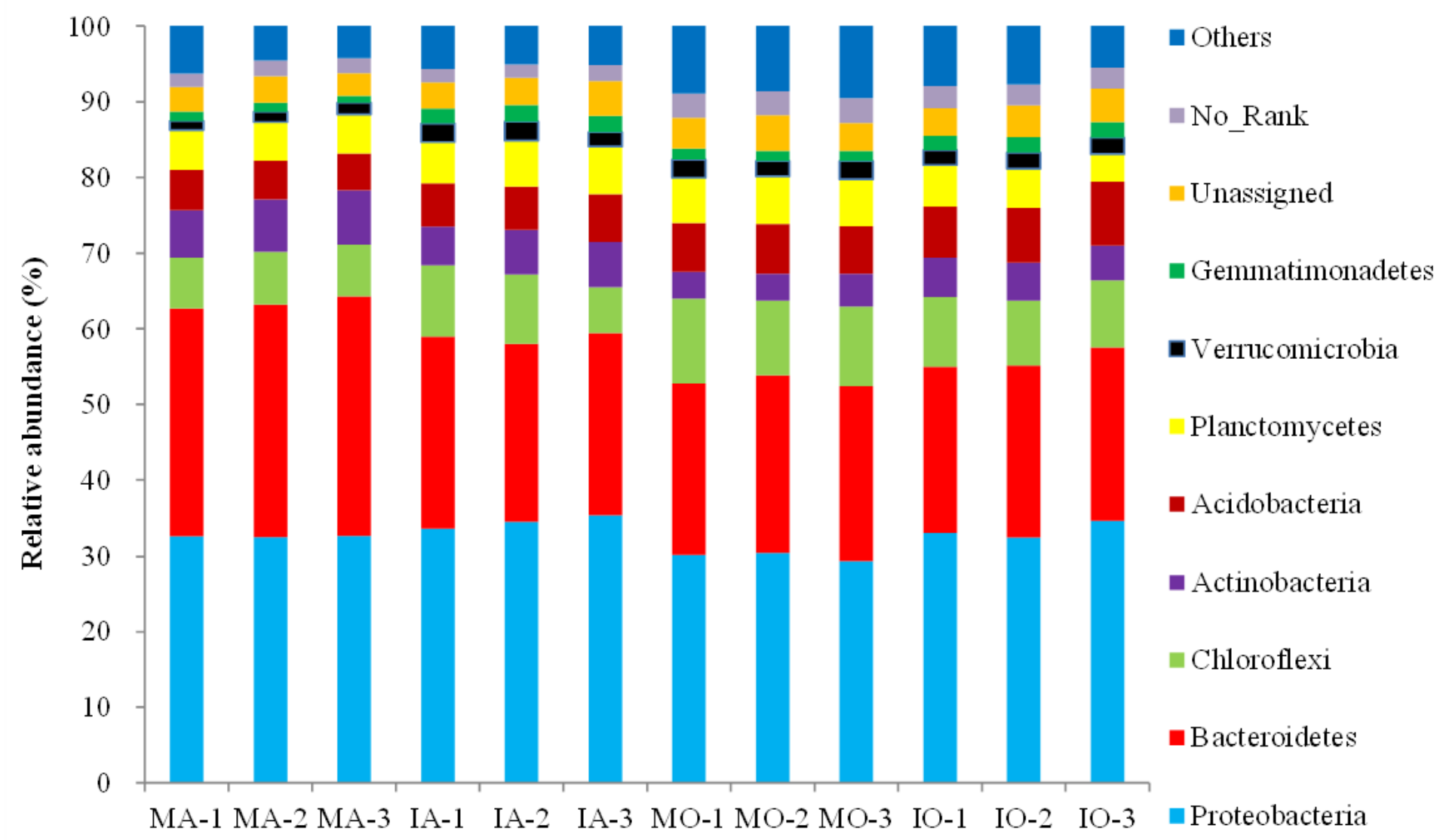

Figure 3

Relative abundance of bacterial phyla for each soil library Note『MA, alfalfa monoculture; IA, intercropping alfalfa; MO, oat monoculture; IO, intercropping oat. 


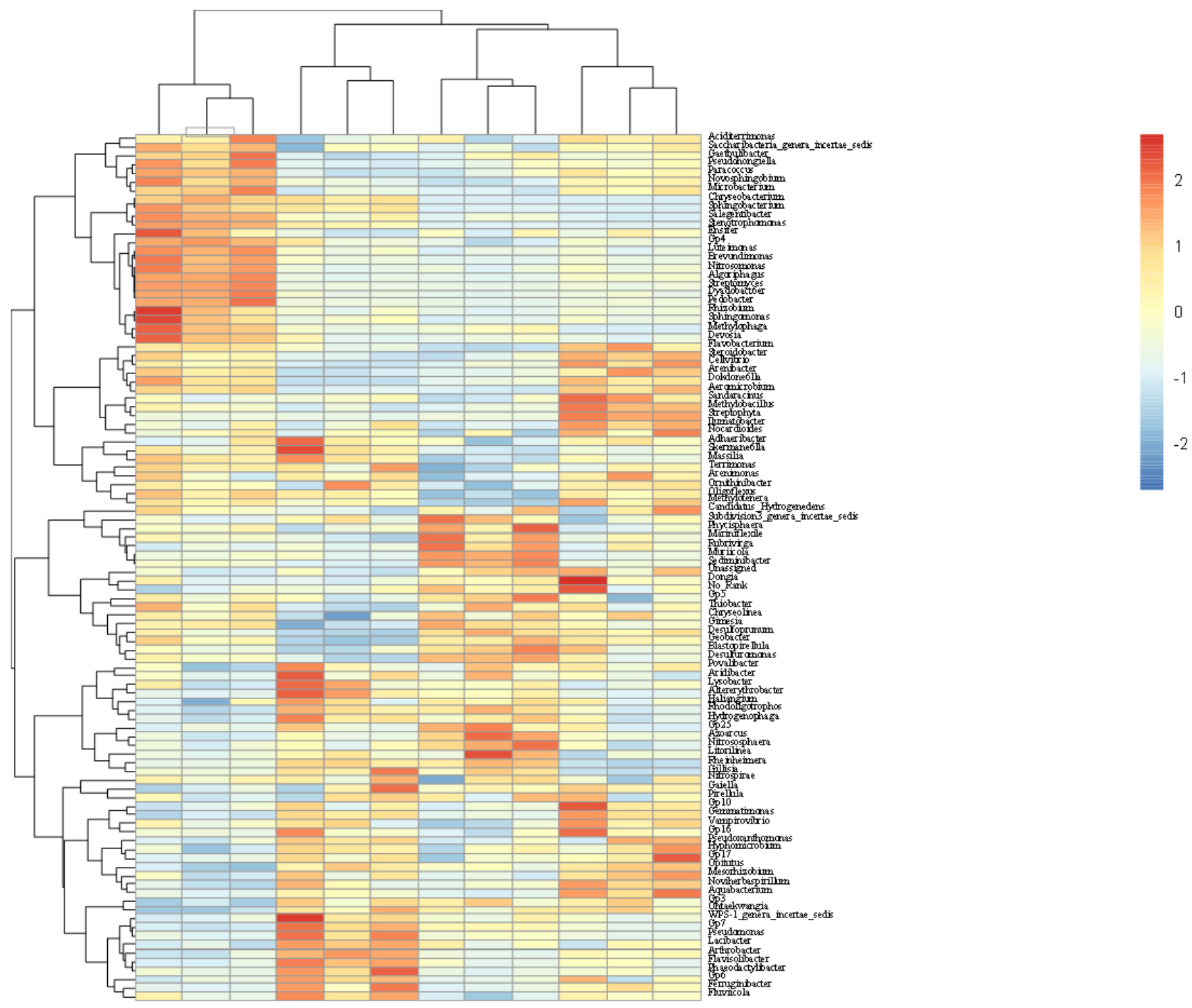

Figure 4

Hierarchical cluster analysis of 100 predominant bacterial communities in different treatments. The OTUs were ordered by genus. Samples communities were clustered based on complete linkage method. The color intensity of scale indicates relative abundance of each OUT read. Relative abundance was defined as the number of sequences affiliated with the OUT divided by the total number of sequences per sample. 


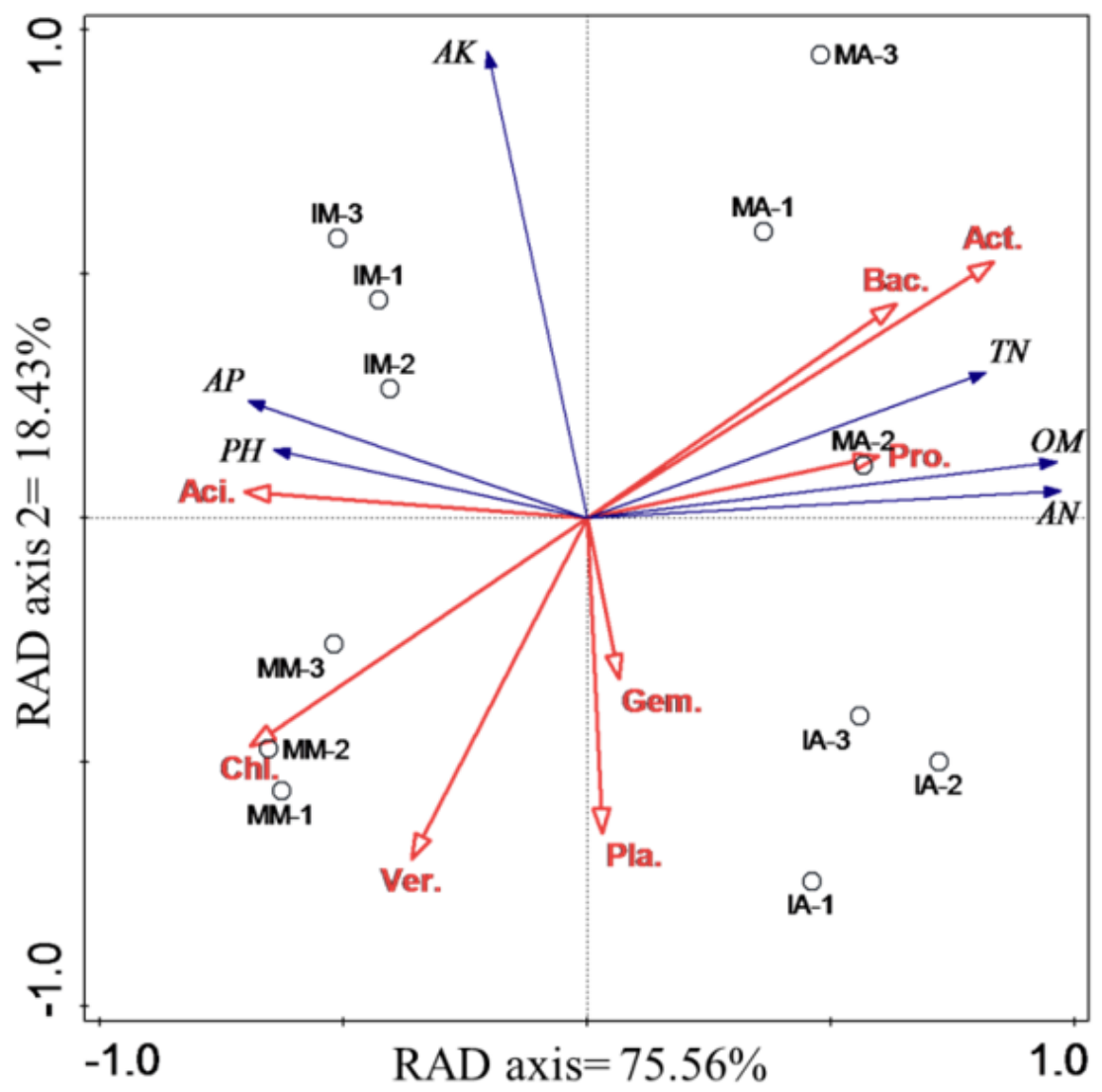

Figure 5

Redundancy analysis (RDA) for soil bacterial community and soil environmental variables. Arrows indicated the direction and magnitude of measurable variables associated with bacterial community structures. RDA component 1 and 2 explained $80.4 \%$ and $11.4 \%$ of the total variations, respectively. Pro. stands for proteobacteria, Bac. for bacteroidetes, Chl. for chloroflexi, Act. for actinobacteria, Aci. for acidobacteria, Pla. for planctomycetes, Ver. for verrucomicrobia, Gem. for gemmatimonadetes, UE for urease, ALP for alkaline phosphatase, CAT for catalase, INV for invertase. 